\title{
Clinical Development of Antiepileptic Drugs in Adults
}

\author{
Bernd Schmidt
}

Neurology \& Psychiatry Clinic, Wittnau, Germany

\begin{abstract}
Summary: Over the last two decades, ten so-called newer antiepileptic drugs (AEDs) have been approved around the world, the majority of which have found and maintained a place in the seizure-fighting armamentarium for the whole spectrum of epilepsies and epileptic syndromes; some of these drugs have features that are improved compared with the older drugs. Within that same time period, the process of clinical development of AEDs has also undergone changes and has become much more complex and costly. Efforts are underway to shift decision-making about the clinical viability of AED candidates to earlier development stages, using the concept of translational medicine. However, thus far all of the newer AEDs have undergone a standard development as an adjunct in the control of adult partial seizures; in some cases, development has been expanded to other seizure types and pediatric syndromes. Cur-
\end{abstract}

rently, the path to global approval for use in monotherapy is under review and is often debated with regulatory authorities. Clinical treatment guidelines consider randomized, blinded, well controlled studies as the premier level of evidence-based medicine; these studies originate mostly in confirmatory phases of the development program. However, with the rigid designs and criteria in regulatory driven trials, effectiveness in the clinical practice setting may not be sufficiently predicted by these studies, leading to frustrations from individual practitioners. Finally, additional safety issues regularly occur only after the postlaunch exposure to a broader population making necessary a continuing, and thorough, pharmacovigilance after the AED has come to market. Key Words: Antiepileptic drugs (AED), clinical trials, adjunctive trials, monotherapy, AED development.

\section{INTRODUCTION}

The first drugs used to treat seizures, bromide and phenobarbital, were discovered purely by clinical observations. Phenytoin was developed preclinically in a rational way by using, for the first time, a maximal electroshock cat model; premarket clinical testing, however, was very limited. In fact, the minimal clinical investigations performed following discovery of preclinical antiseizure activity of all of the older antiepileptic drugs (AEDs; drugs that that are still frequently used, such as carbamazepine or valproate) would not satisfy regulators for marketing authorization today. Antiepileptic drugs coming to market after valproate are frequently referred to as "newer" AEDs, even though some already have become generic. In recent years, the clinical development process of AEDs has become increasingly regulated and supervised in the U.S. by the Food and Drug Administration (FDA) and in Europe by the European Agency for the Evaluation of Medicinal Products

Address correspondence and requests for reprints to: Bernd Schmidt, M.D., Ph.D., Neurology \& Psychiatry Clinic, Hasenbuckweg 14, D-79299 Wittnau/Germany. E-mail: drs.schmidt.wittnau@t-online.de.
(EMEA). This process has become more methodologically complex, and with this, more expensive. ${ }^{1}$

\section{THE TRADITIONAL DEVELOPMENT PROCESS}

The clinical development of AEDs follows, in general, the pathway established for all new chemical entities. The process begins with a first application in man in phase-I, progresses through preliminary establishment of efficacy and tolerability and short-term safety in phaseII, followed by confirmation of the emerging effectiveness profile in larger scale studies in phase-III. All this must be accomplished before a new drug application or application for market authorization is filed (or equivalent) in the various regions and countries around the world. Phase-IIIb studies, typically published shortly after the new chemical entity is approved, and phase-IV studies, performed after the drug is on the market, are intended to broaden the knowledge about the new medication in a wider population and in a practice-oriented setting. As noted above, this process is highly regulated and supervised. Before an experimental drug is permitted to be given to a human being, a full dossier on preclinical 
effects as a potential AED and preclinical safety pharmacology and toxicology are reviewed by the government authorities and institutional ethics review boards. ${ }^{2,3}$ An investigational new drug application is granted only when the risk-benefit analysis is found to be favorable.

Typically, phase-I trials are performed in special clinical research institutions. Single rising doses are applied in the first study to healthy male volunteers, sometimes including women of non-childbearing potential, in order to establish a maximum-tolerated dose and the profile of adverse events (AEs) in healthy subjects, as well as to obtain human pharmacokinetics and metabolism data. A forerunner model takes care that not too many subjects are exposed to a novel compound at the same time.

After a maximum-tolerated dose in single application is established, a larger study in healthy volunteers is initiated to explore tolerability and short-term safety with multiple dosing, often over two to three weeks, again in a rising dose design, with various cohorts. Dosing and dose intervals will be modeled after the pharmacokinetic data obtained in the previous single dose study. A detailed analysis of disposition parameters and metabolism is performed, and special tests for cognition as well as cardiac and electroencephalographic parameters may be added.

Results from single and multiple dose studies may make it necessary to change the current formulation, e.g., if peak level dose toxicity occurs; this might need to be accomplished before going to patients. In an extreme case, the project may have to be terminated if either the unfavorable pharmacokinetic or metabolic features do not fit the desired drug target profile. In addition, if the margin between the predicted therapeutic plasma concentrations and the observed values in volunteers is too narrow, the drug may be abandoned.

On the other hand, tolerability in healthy volunteers may not directly reflect tolerability in epilepsy patients as pharmacoresistant patients often adapt to typical CNS AEs from AEDs better than their healthy counterparts. Sometimes, due to the in-house setting of phase-I units and the background of the volunteers in early phase-I studies, categories of AEs are reported that tend to be artificial; these AEs may not reappear in the later clinical therapeutic studies in patients, especially in the area of mood AEs. Studies in phase-I in volunteers also do not provide information about tolerability and safety of AED combinations. This issue becomes very important in early phase-II, as the experimental new drug will be added to an existing regimen in subsequent patient trials.

Depending on those initial results, a whole series of phase-I studies with pharmacokinetic or pharmacodynamic questions are next initiated in special populations. These small but important studies are typically concomitant with the ongoing clinical therapeutic trials and sometimes total as many as 15 to 20 studies in the final dossier. As an aside, problems in interpretation of phase-I studies accumulate when too many answers are demanded from one study.

In phase-II, the experimental potential AED is applied to patients with epilepsy for the first time. Traditionally, in phase-IIa a limited number of patients with medication-resistant and frequent partial seizures receive the novel compound on top of their stable baseline AED medications in a controlled, blinded design. The aim is to get information about the maximum tolerated dose in the population of interest, obtain pharmacokinetic and metabolism data in patients, learn about potential pharmacodynamic and pharmacokinetic interactions with other AEDs in a clinical environment, and, finally, obtain preliminary indication on antiseizure activity. Phase-IIb, which follows, uses the data from IIa, and includes more patients, typically in one or more randomized, parallelgroup studies with various dosages and sufficient duration of exposure. The object is to define the dose-response curve of a potential AED; i.e., to discern between a minimally effective dose and a poorly tolerated dose, again under the conditions where the novel drug is added to an existing AED regimen that did not control the patients seizure activity in the past. ${ }^{4,5}$ Crossover trials, included in the package for some of the earlier "new" AEDs, presently are not acceptable to the FDA or the EMEA as pivotal evidence because of methodological limitations. However, under certain circumstances, well planned crossover designs may be useful within the context of early clinical exploratory work. ${ }^{6}$

One classic mistake, frequently observed in AED development in the past few decades, is the underestimation of the value of a solid phase-II program. This has led to inaccurate dosing and titration regimens when entering the later pivotal studies, which then yield an unfavorable effectiveness pattern in the late stages of development-a major problem.

For obtaining marketing approval, aside from providing a good body of evidence that the novel AED is well tolerated and safe in both short- and middle-term use in the targeted population, each efficacy endpoint needs to be confirmed and replicated in at least two pivotal studies of sufficient duration in a phase-III program. As these studies are extremely costly, they are designed in a way to satisfy regulatory authorities in the U.S. and Europe, as well as the other regions in the world, if possible. Japan, even though part of the International Conference on Harmonization of the Technical Requirements for the Registration of Pharmaceuticals for Human Use effort, still follows its own guidelines with the result that novel AEDs are either approved with considerable delay or never at all. ${ }^{7,8}$

Phase-IIIb trials, initiated before approval and frequently published after the novel AED has been launched, can be less rigid in design. These studies do 
provide valuable information related to the optimal use of the drug, if properly designed. Also, phase-IV studies initiated after launch can be informative, especially regarding the effectiveness in a broad population and the best dose regimen; data on extended exposure and pharmacovigilance may also be obtained.

Within the traditional drug development process that AED researchers have followed over the past two decades, approvals were first sought for adjunctive treatment of partial seizures in adults (whether or not the attacks were secondarily generalized), followed by adjunctive treatment of primary generalized tonic-clonic seizures in adults and then extending to adjunctive treatment of pediatric partial seizures. Then, depending on the profile of the drug, further filings for monotherapy in partial seizures and special syndromes (e.g., LennoxGastaut syndrome or myoclonic epilepsy) were added. ${ }^{9}$

\section{NOVEL TRENDS AND CHANGING PARADIGMS}

As with new chemical entities in most therapeutic areas, clinical development of AEDs has become, over the years, increasingly complex and therefore more costly. There has been an appeal by pharmaceutical companies for reduced development times; this would not only decrease development costs, but would also extend the life cycle of a compound before it becomes generic. In addition, in an attempt to minimize the risk of a failed development in the expensive later development stages, emphasis has been placed on methods and criteria either to reliably evaluate signals of anticonvulsant potential in humans or to terminate the program as early as possible.

The concept of translational medicine has entered AED development in the search for adequate biomarkers. These, after being validated, may be used in a small number of patients to predict the degrees of the desired response, unwanted toxicity, or metabolic features. The attempt to develop human biomarkers that are sensitive to change by potential anticonvulsants is, in part driven by the observation that novel drug targets may be missed by the current routine anticonvulsant screening programs in animals. Pharmacokinetic pharmacogenomics and pharmacodynamic pharmacogenomics are currently areas of focused research for relevant biomarkers; in addition, imaging techniques can be used for exploration of epileptogenesis in human brain tissue. ${ }^{10,11}$

Currently, in AED development in adults, proof-ofconcept (PoC) or proof-of-principle (PoP) studies serve the purpose of gaining early confidence in a drug's anticonvulsant activity in humans. Within the traditional development sequence, the PoC study is placed, depending on the individual profile of the drug, after the single or multiple dose phase-I study; it may be the only phaseIIa study performed.
With the increasing importance of PoP as critical for decision making, requirements for an optimized lead compound to be tested in a PoP study are upgraded as well. Strong support of the approached indication has to be available in a whole range of suitable in vitro and animal models; dose responses must be thoroughly investigated. Efficacious concentrations and pharmacokinetic behavior of the compound in humans has to be reasonably predicted; metabolic profiling must also be performed. Both preclinically and in human volunteers, toxicologic liabilities and a potential therapeutic window need to be determined. Single and possibly multiple dose toxicology studies, including maximal tolerated doses, need to have been undertaken in healthy volunteers to support short-term application in a suitable PoP human population. An often overlooked requirement is that formulations that achieve efficacious concentrations without too much variability in plasma and the central nervous system compartment are available and can be manufactured to good manufacturing standards.

Typically, a handful of $\mathrm{PoC}$ models have been used for candidate AEDs. They can be grouped into those models that use seizure counts as the clinical endpoint and those models with an electrophysiological outcome measure. Because of the nature of epilepsy, the evaluation of seizure counts requires chronic toxicology studies to allow for longer observation periods. When using surrogates, however, single dose applications may be sufficient to achieve a good result. The photosensitive human model using the reduction or elimination of a photoparoxysmal response as a surrogate has been successfully used as $\mathrm{PoC}$ in recent development programs. ${ }^{12}$

Given the pressure to make early decisions and to leap into pivotal studies in a novel concept of "learn and confirm"-rather than the conventional but slower phase-I to phase-III approach-there is a danger that the dose exploration, assigned to phase-II, will not be done in as extensive and widespread a fashion as necessary. The consequence may be a poor understanding of the dose range when the drug reaches the market. This is even more relevant when new AEDs need to be titrated; in such cases, if the optimal titration scheme is not defined before entering the pivotal part of the program, the results may be uninterpretable. Sometimes phase-II and phase-III studies are amalgamated to create mega-trials, which can present a special set of problems. ${ }^{13}$

Instead of approaching a larger market by receiving approval for an indication for treatment of partial seizures first and then expanding into other indications afterwards, filing initially for an orphan drug niche (e.g., Lennox-Gastaut syndrome) has become an alternative strategy for marketing.

A thus far unreached goal for future developments of AEDs would be demonstration of a true disease-modi- 
fying effect on top of the antiseizure activity. Such demonstration will require suitable trial designs.

\section{ADJUNCTIVE TRIALS}

Due to ethical concerns and effective regulatory guidelines, all AED development programs begin their clinical development by adding the experimental anticonvulsant to an existing baseline AED regimen. By nature, this procedure puts the new drug at a disadvantage, as patients recruited for those trials are already partly treated by one to four AEDs but remain uncontrolled. The new compound must therefore demonstrate an incremental benefit, when compared with a control group. In adjunctive use, adverse pharmacodynamic effects may accumulate more than in monotherapy, and pharmacokinetic interactions with baseline medications are a major issue. ${ }^{14}$

The first market license is usually granted for treatment of partial seizures and is based on pivotal studies, including, typically, a population of uncontrolled patients with a minimum mean baseline seizure frequency of four partial seizures per month (in essence, complex partial seizures). The patients are within the legal age range, typically between 18 and 65 years. Standard protocols require more or less "otherwise healthy" patients with a low number of allowed comedications. This rigidity of inclusion and exclusion criteria does limit extrapolation of the effectiveness of a novel AED from data contained in the registration dossier to a wide clinical use in a broad population after launch.

Phase-II and confirmative phase-III trials aimed at a demonstration of efficacy, tolerability, and safety must be well controlled and blinded. In epilepsy, fluctuations of seizure patterns are well known, and even in patients considered pharmacoresistant, a variable placebo effect is documented. Therefore, the design of choice for adjunctive trials includes the following: 1) a multiarm, parallel group, 2) placebo control, 3) a prospective baseline, and 4) a titration period of adequate length. The latter depends on the pharmacokinetic features of the compound and previous tolerability information. In addition to the above, a maintenance phase of at least three months and a tapering period are required. Finally, a blinded transition period is needed for those patients who elect continuation into an open label extension protocol. ${ }^{15}$

In general, phase-IIb dose ranging studies consist of at least three active arms versus placebo. These studies are add-on and seek superiority of at least one of the doses compared with placebo treatment. Ideally, if the doses are correctly chosen, a dose-response curve will provide both a minimally effective and a maximum-tolerated dose level. In adjunctive treatment trials, an active comparator "gold standard" as calibrator may provide early useful clinical comparative information; such compara- tors are usually not included, however, because of the added complexity of the design.

In reality, two strategies have been employed in the later stages of development of an AED in adults. The first is to go forward immediately after $\mathrm{PoC}$ with a large-scale phase-II study with up to six arms using all the design features of a pivotal study and then picking the best dose(s) for confirmation in two, mostly geographically separated, two-arm phase-III studies. The second approach is to perform limited dose ranging phase-II work, investing later in at least two larger confirmatory studies; these studies will require overlapping dosing patterns between the studies. Adaptive designs can compromise between the two strategies and, if well conducted, might save development time.

Depending on the potency of the novel AED, sample size calculation for pivotal parallel group adjunctive studies of three-month duration typically require 60 to120 patients. In the AED-refractory partial epilepsy populations in phase-IIb/III trials, those patients who have failed at least two optimally dosed AED regimens in the past usually have baseline seizure frequencies in the range of 9-12 partial seizures per month. Partly because of this observation, prospective baseline periods, formerly three months long, have been shortened and are now mostly between six and eight weeks in duration. ${ }^{7,8}$

As there is no disease-modifying AED to date, reduction of seizure frequency is the primary measure of efficacy. Capturing, handling, and analyzing seizure data are far from easy. Seizure data are recorded by the patient or caregiver on paper or, more recently, by electronic diaries; either method serves as the source data. For the purpose of a trial, a personal coding system may be agreed upon between the patient and the investigator for describing various multiple seizure types as they occur; it remains, however, the responsibility of the investigator to accurately transfer the patient's own observations into the International Classification of Seizures on a case record form.

In an adjunctive AED development program, the main and first-approached target indication is treatment of partial seizures. Each seizure type is recorded and evaluated separately; however, the vast majority of seizures counted are complex partial seizures. Simple partial seizures, which may be auras, are sometimes difficult to differentiate; therefore, under clinical trial conditions, only those partial seizures with a recognizable motor component are included. In some trials, auras are completely excluded. For the older drugs and for the older of the newer drugs, labels did include secondarily generalized partial seizures (SGTC) if a subpopulation with this seizure type had been recruited in pivotal trials. Unfortunately, the views of the regulatory bodies have changed. Now it is usually necessary to demonstrate an effect on this propagating seizure type by well powered 
studies, independent of any proven effect on partial seizures themselves.

Two principal standard outcome variables are used in adjunctive therapeutic AED trials: 1) median change of seizure frequency compared with baseline, and 2) the categoric outcome of percentage of patients with a reduction of seizure frequency by at least $50 \%$ from baseline value. Comparison is then always made between the endpoints of the active dosage group versus the placeboadded group. Median absolute or relative change to baseline has been favored by individual countries and the EMEA, while the dichotomous outcome has been favored by the FDA in the past. ${ }^{16}$

The clinical relevance of any certain reduction in seizure frequency is debatable, and seizure freedom for the duration of the study has been proposed as the most relevant outcome. However, despite the flurry of newer AEDs coming to market, none has convincingly been able to increase the population of pharmacoresistant partial epilepsy patients who are seizure-free over prolonged periods.

Seizures are distributed in a nonparametric manner. When handling and analyzing incoming seizure records, this fact needs to be taken into account by the statistical procedures and tools applied; e.g., by normalizing the data. One attempt to deal with the skewed distribution has been the introduction of a response ratio (RR), defined by the difference between baseline and seizure frequency with treatment, divided by the sum of baseline plus treatment seizure frequency. ${ }^{7,8}$ A RR of -0.33 does correspond to a $50 \%$ reduction from baseline, as traditionally displayed. Great care needs to be taken when comparing results among studies. This is especially important when performing a meta-analysis without knowing exactly what kind of path the raw data have followed through the statistical analyses. In any case, the statistical plan needs to be defined a priori before the blind of a trial is broken.

Pivotal studies are primarily evaluated for registration purposes following the intent-to-treat (ITT) principle. Here, all patients randomized are included, assuming receipt of at least one application of the test article and one outcome measurement. As a standard, within the ITT, the last value obtained is taken as the final outcome and is included in the analysis if the patient drops from the study. This is called the last observation carried forward method. This method, unfortunately, may lead to considerable artifacts in terms of judgment of true effectiveness of a new medication, especially if dropouts occur early in the treatment phase; mostly due to tolerability issues. These dropouts typically arise from the use of a too-rapid titration scheme or a too-high fixed dose in one or more of the blinded treatment arms. Consequently, with strict ITT, the response on seizure rate at endpoint could theoretically be lower at a toxic, but effective high dose of an experimental AED, compared with a well working and tolerated middle dose. In such a case, many patients drop from the high dose arm very early, their still-high seizure rates are carried forward, and at the endpoint, looking over the treatment arms from a low to a high dose, an inverse bell curve is mimicked. ${ }^{17}$ To avoid these artifacts, a number of "modified ITT" versions have been introduced which censor data at time of the dropout. In addition, care should be taken to understand, from the results presented, whether the analysis includes a titration period or only refers to a maintenance period. When it comes to prolonged titration periods, the protocol should clearly state exactly how the titration was applied among the treatment groups and how dropouts within the titration period have been handled in the analysis. With prolonged titration phases it is quite possible that efficacious doses are achieved in one treatment arm in a shorter time than in one or more of the other arms. The statistical study evaluation plan must specify, in advance of analysis, how to handle seizure clusters, missing data in non-dropout patients, reduced or missed doses, and serious noncompliance.

The per protocol analysis only includes those patients in full compliance with the protocol requirements; patients who have tolerated the foreseen exposure and reached the endpoint. These patients provide a picture of efficacy in those patients tolerating the AED at the assigned doses. Responder rates are overestimated in the high dose groups if a great number of patients leave the study due to intolerable AEs. ${ }^{18}$

In an analysis of adjunctive partial seizure trials, in addition to providing data on the total seizure count in each of the blinded treatment arms versus the placebo arm, a breakdown of the seizure types needs to be displayed. Aside from the sheer total numbers, it is valuable clinical information if there might be a desired shift to less severe types; e.g., loss of secondary generalization. Likewise, one should know if the undesired induction of new seizure types occurred; e.g., a shift from simple partial to SGTC seizures without a warning aura.

For standard primary variables such as the mean reduction or $50 \%$ responder rate, impressively low $P$ values in group comparisons versus placebo or between different development programs must always be evaluated against the clinical relevance of the overall results in terms of effectiveness. A highly statistically different result versus placebo in adjunctive treatment may be favored by a number of design and population factors, including an overpowered trial.

Secondary variables of adjunctive AED trials in adults include rating and scales on seizure severity, neurotoxicity, and quality of life; e.g., the Quality of Life in Epilepsy instrument. These and other patient-related outcomes, however important, cannot stand alone if there is 
no substantial reduction in the individual patients' seizure activity and, as the ultimate goal, seizure freedom. ${ }^{19}$

Long-term continuation studies, following the registration phase-III trials and newly initiated phase-III and postmarketing phase-IV studies, use retention time as a composite marker of effectiveness over extended periods under clinical field conditions and provide more flexibility in treatment paradigms. ${ }^{20}$

The safety database in a registration dossier for a novel AED contains between 1200 and 2000 volunteers and patients, from single doses in phase-I up to the large confirmatory studies with extensions and separate openlabel safety trials. When other CNS indications are applied for at the same time in the same package, the safety pool may go up to about 4000 individuals. Regulations call for the inclusion of special populations, such as elderly and pediatrics, but depending on the emerging profile of the drug other special risk groups need to be explored.

Antiepileptic drug treatment is chronic treatment, mostly lifelong. Tolerability of a new AED is established relatively early in the development program. The pattern emerges in phase-I and phase-II; this pattern depends on the formulations used, the titration schemes, and the maximal tolerated doses. When entering large confirmative studies, measures to optimize tolerability should have been taken into account. However, with the short duration of three-month controlled trials, safety signals are easily overlooked or are not obvious because of their cumulative nature. In a registration dossier, the number of patients continuously exposed to the new AED over many years with adequate doses is usually quite low. In addition, in a refractory epilepsy population on polymedication, safety signals may be easily masked or misinterpreted. In the past, serious idiosyncratic reactions and cumulative toxicity of a compound previously believed to be safe have been realized only after long years of marketing. ${ }^{21}$

To address some of the potential issues, the clinical pharmacology package of a dossier does include several studies; e.g., on potential prolongation of cardiac QT intervals or exposure of organ failure patients. This package also addresses the special features a drug may have; e.g., on cognition or in the elderly, or through collection of prospective data in a pregnancy registry.

It has been well noted that incidence of AEs, of a subjective nature in particular, vary considerably between investigational sites and regions. The U.S tends to report AEs with more frequency than Europe, and both report more than Japan with the same drug and dosing. When comparing AE profiles among sites, incidences may differ due to the inclusion of "recycled" patients in a research-type clinic versus patients who are in their very first AED trial—in a more practice-oriented setting.
Adjunctive trials have been carried out in primary generalized tonic-clonic seizures (PGTC), LennoxGastaut syndrome (LGS), and myoclonic epilepsy, with at least a proportion of the population being adults. In PGTC trials, issues include the correct diagnosis of this seizure type within generalized epilepsy versus SGTC seizures. With LGS the issues are multiple, beginning with the transatlantic controversy about diagnosis, the definition of a relevant composite endpoint (e.g., "major motor seizures"), and ways of correct seizure counts and data handling when seizure frequency is reported, sometimes up to amazingly high figures. ${ }^{9}$

\section{MONOTHERAPY TRIALS}

As in clinical practice, in a clinical AED development program monotherapy can be achieved through three different routes: down-titrating comedicated AEDs from a previous polytherapy regimen, switching from one monotherapy to another via a short adjunctive period, or introducing the new AED de novo to drug naïve patients. However, in contrast to the fairly straightforward approach used to demonstrate a statistical superiority of the experimental AED versus placebo in an add-on development program, the regulatory path for approval of an AED as a single drug has, in recent years, become controversial. This controversy has led to a deadlock in terms of finding one global monotherapy program that will lead to approvals with the EMEA and the FDA on the same terms. ${ }^{22}$

In the US, on the back of a solid adjunctive dossier for the same drug, a license for withdrawal to monotherapy in refractory patients has been granted based on an outpatient conversion to monotherapy trial designs, in which patients stabilized on an adjunctive regimen were randomized to receive either the experimental AED, a placebo, or a low dose active compound meant to be a pseudo-placebo. Then the baseline AED(s) were slowly withdrawn. Escape criteria categorize the patients as "successes" or "failures." In the case of a statistically significant difference between the two groups in favor of patients on full dose monotherapy, the trial is considered positive. Criticism includes ethical concerns about the use of a noneffective treatment arm in monotherapy and the fact that the new AED is tested for activity in a labile, withdrawal paradigm only. ${ }^{23}$

Inpatient presurgical withdrawal designs may be suitable for PoC, but have been found to be obsolete for registration purposes. Concerns include the short duration time of a few days and the lack of generalizability of the results to a general partial epilepsy population, as well as ethical issues. ${ }^{24}$

To support a label to treat seizures in monotherapy in newly or recently diagnosed epilepsy, some patient study designs feature time to $\mathrm{n}^{\text {th }}$-seizure and seizure-free peri- 
ods at, e.g., six months and one year as endpoints, seeking superiority of one active full dose arm treatment arm over a control. In one variation of that method, patients with frequent partial seizures received blinded and randomized amounts of the experimental AED in therapeutic doses, versus the other group, which received placebo. The trial was of short duration, as the active arm separated rapidly and showed a high statistical difference from placebo, as expected. However, ethical issues on the use of placebo in such an experimental monotherapy situation remain. ${ }^{25}$ Another variation of the newly diagnosed monotherapy design aimed at demonstrating a difference is to recruit a more generalized population with fewer seizures since diagnosis (and either no treatment or no chronic treatment) and randomize the patients into two groups: a good dose and a dose thought to be marginally effective (if effective at all). The analysis uses the time to $\mathrm{n}^{\text {th }}$-seizure as the primary outcome variable. Study duration is individualized and may continue up to 2.5 years. Seizure-free time is a clinically relevant secondary measure. There are a number of problems involved in this design as well, beginning with selection of the right patient population, selection of doses, suitable titration regimen, and again ethical concerns about using an a priori intended inferior dose for comparison. ${ }^{26}$ In an effort to overcome the current uncertainties about how to approach a monotherapy indication in the U.S., the idea of using a historical active control group as an anchor for results obtained by a novel AED has recently been proposed to the FDA.

The EMEA, in contrast to the FDA, does accept active comparator noninferiority trials as pivotal for approval of a monotherapy indication, provided the noninferiority margin is acceptable and the study is powered accordingly. Most of the studies performed thus far have used carbamazepine as a "gold standard" reference for partial seizures. The response of the FDA to noninferiority designs has not been favorable, as assay sensitivity and intrinsic validity need to be demonstrated for each new trial. When designing clinically informative head-tohead studies, care should be taken to design the study in a fair way between the new AED to be tested and the active comparator, especially in terms of the titration scheme, the full maintenance dose, the formulations used, and the procedures for conditioned dose adaptation.

\section{FROM REGULATORY TRIALS TO USE IN CLINICAL PRACTICE}

The transition from the clinical information gained in a standard formal AED development program to the hands-on clinical experience after the AED has been marketed often reminds one of a green banana reaching the customer. This is due to a number of factors. Under regulatory driven clinical trial conditions, patients are selected in such a way to be as homogeneous as possible. Then, for example, in adjunctive partial epilepsy studies, the patients are forced to titrate the experimental AED within a rigid schedule to fixed preassigned doses. If either the titration or the top dose leads to intolerance, patients drop out. Using the ITT analysis, their last observed seizure count is added to the dose group of those patients who complete the study as planned.

In clinical practice, choice of an AED is targeted to the individual patient's overall condition, age, gender, social situation, profession, mental ability, and multiple other factors, taking into account the perception of an AED in general. Uptitration is done slowly and, depending on the patient's report on subjective AEs, with variable length related to the dose increments and the time between dose increase steps. As the goal is seizure freedom, doses are increased until either patients are controlled or tolerability and safety concerns limit further dose increases. Individual optimization of a new AED therapy in clinical practice also may include reduction, withdrawal, or addition of baseline AEDs, a procedure that might be supported by plasma level monitoring.

Comparatively high responder rates in adjunctive trials, and a high incidence of seizure-free patients in adjunctive and monotherapy trials, are strong predictors of a novel AED performing well in terms of efficacy in clinical practice, as those rates tend to be even higher when less severely afflicted patients are treated in clinical practice with their individual conditions taken into account. Recommended titration schemes, starting doses, and target doses that are derived from a clinical development program of AEDs in adults may not do well when transferred to a generalized clinical practice. Titration, if recommended at all, is usually too rushed and the starting dose is often too high, as is the median dose for a typical patient. However, the contrary may also happen when doses too low for a maximized efficacy have been explored in the development program.

Surprises are not rare after a novel AED has come to the market. These may come from unexpected AEs when a broader range of the epilepsy population is exposed to the new AED for extended periods. Tolerance to efficacy may be observed as may worsening of seizure types not considered in the development program. Further, the occurrence of rare but serious idiosyncratic reactions may appear. All those factors underline the importance of phase-IIIb and phase-IV studies, as well as targeted registries; e.g., to document potential teratogenicity in humans.

Overall, in clinical trial developments aimed at registration of an AED for marketing, efficacy, tolerability, and safety are explored under standardized and somehow artificial conditions. In clinical practice, effectiveness is what counts. A composite of factors that suits the indi- 
vidual patient best are most important when using the novel AED. Treatment guidelines for epilepsy have to take into account results from well controlled regulatory clinical trials as part of evidence-based medicine, but must also consider postmarketing effectiveness and head-to-head studies in a clinical practice environment, as they become available over time. ${ }^{1,4}$

\section{REFERENCES}

1. Katz R. FDA update. Epilepsy Res 2006;68:85-94.

2. White S. Preclinical development of antiepileptic drugs: past, present and future directions. Epilepsia 2003;44(suppl 7):2-8.

3. Bickerstaffe R, Brock P, Husson J-M, et al. Ethics and pharmaceutical medicine. Int J Clin Pract 2006;60:242-252.

4. Schmidt B. Gabapentin. In: Levy R, Mattson R, Meldrum B, Penry I, Dreifuss FE, eds. Antiepileptic drugs. 3rd ed. New York: Raven Press; 1989:chap 68.

5. Wolf P, Schmidt B. Early phase II testing of ralitoline. Excerpta Medica 1988:ISBN 444-01365-2:45-56.

6. Messenheimer J, Ramsay RE, Willmore LJ, et al. Lamotrigine therapy for partial seizures: a multicenter, placebo-controlled, double-blind, cross-over trial. Epilepsia 1994;35:113-121.

7. Gabapentin in partial epilepsy. UK Gabapentin Study Group. Lancet 1990;335:1114-1117.

8. Gabapentin as add-on therapy in refractory partial epilepsy: a double-blind, placebo-controlled, parallel-group study. The US Gabapentin Study Group No. 5. Neurology 1993;43:2292-2298.

9. Sachdeo RC, Glauser TA, Ritter F, Reife R, Lim P, Pledger G. A double-blind, randomized trial of topiramate in Lennox-Gastaut syndrome. Topiramate YL Study Group. Neurology 1999;52: 1882-1887.

10. Littman BH, Williams SA. The ultimate model organism: progress in experimental medicine. Nat Rev Drug Discov 2005;4:631-638.

11. Katz R. Biomarkers and surrogate markers: an FDA perspective. NeuroRx 2004;1:189-195.

12. Schmidt B. Proof of principle studies. Epilepsy Res 2006;68:48-52.

13. Schmidt B. How to avoid misinterpreting results of clinical trials. Epilepsia 2005;46(suppl 6):18.
14. Benedetti MS. Enzyme induction and inhibition by new antiepileptic drugs: a review of human studies. Fundam Clin Pharmacol 2000;14:301-319.

15. Burneo JG, Montori VM, Faught E. Placebo effect in antiepileptic drug trials. Epilepsy Behav 2003;4:371.

16. Pledger GW, Sahlroot JT. Alternative analyses for antiepileptic drug trials. Epilepsy Res Suppl 1993;10:167-174.

17. Barcs G, Walker EB, Elger CE, et al. Oxcarbazepine placebocontrolled, dose-ranging trial in refractory partial epilepsy. Epilepsia 2000;41:1597-1607.

18. Schmidt B. Randomized monotherapy trials of new anti-epileptic drugs. Can "evidence-based medicine" be misleading? Epilepsia 2002;43(suppl 8):21-22.

19. Baker GA, Currie NJ, Light MJ, et al. The effects of adjunctive topiramate therapy on seizure severity and health-related quality of life in patients with refractory epilepsy - a Canadian study. Seizure 2002;11:6-15.

20. Ben-Menachem E, Edrich P, Van Vleyman B, Sander JWAS, Schmidt B. Evidence for sustained efficacy of levetiracetam as add-on epilepsy therapy. Epilepsy Res 2003;53:57-64.

21. Wong ICK, Lhatoo SD. Adverse reactions to new anticonvulsant drugs. Drug Saf 2000;23:35-56.

22. French JA, Schachter S. A Workshop on AED monotherapy. Epilepsia 2002;43(suppl 10):3-27.

23. Beydoun A, Fischer J, Labar DR, et al. Gabapentin monotherapy: II. A 26-week, double-blind, dose-controlled, multicenter study of conversion from polytherapy in outpatients with refractory complex partial or secondarily generalized seizures. The US Gabapentin Study Group 82/83. Neurology 1997;49:746-752.

24. Bergey GK, Morris HH, Rosenfeld W, et al. Gabapentin monotherapy: I. An 8-day, double-blind, dose-controlled, multicenter study in hospitalized patients with refractory complex partial or secondarily generalized seizures. The US Gabapentin Study Group 88/89. Neurology 1997;49:739-745.

25. Schachter SC, Vazquez B, Fisher RS, et al. Oxcarbazepine: double-blind, randomized, placebo-control, monotherapy trial for partial seizures. Neurology 1999;52:732-737.

26. Arroyo S, Dodson WE, Privitera MD, et al. Randomized dosecontrolled study of topiramate as first-line therapy in epilepsy. Acta Neurol Scand 2005;112:214-222. 